\title{
LA REVISTA DE MEDICINA Y CINE COMO FACILITADOR DE PUBLICACIONES CIENTÍFICAS DE LOS ESTUDIANTES
}

\section{The Journal of Medicine and Movies as a facilitator of students' scientific publications}

Jorge PÉREZ1; ; María José FRESNADILLO²; José Elías GARCÍA-SÁNCHEZ²; Enrique GARCÍA-SANCHEZ²

${ }^{1}$ Facultad de Ciencias de la Salud y de la Vida. Universidad Pompeu Fabra. Barcelona (España). ${ }^{2}$ Facultad de Medicina. Universidad de Salamanca (España).

e-mail: jordi.perez@upf.edu

Fecha de recepción: 5 de abril de 2019

Fecha de aceptación: 23 de mayo de 2019

Fecha de publicación: 15 de marzo de 2020

\section{Resumen}

La Revista de Medicina y Cine siempre estuvo abierta a publicar artículos de alumnos tanto de ciencias de la salud como de otras titulaciones. Una de las pretensiones de la revista fue el estimular la participación de los estudiantes como autores de sus publicaciones. El objetivo del presente artículo consiste en describir la participación de estudiantes en la revista informando del número de artículos publicados, del número de autores, del tipo de trabajo realizado, de las universidades de procedencia, de las películas comentadas y de los temas tratados.

Se revisaron todos los artículos publicados desde el año 2005, inicio de la revista, hasta el año 2019. Se registraron 42 artículos con participación de estudiantes (16,9\% del total) con 60 autores procedentes de 12 universidades distintas y de 10 titulaciones diferentes, seis de ellas de ciencias de la salud.

Se concluye que la Revista de Medicina y Cine, además de ser un medio para fomentar el aprendizaje en estudios de ciencias de la salud, puede facilitar a los estudiantes el desarrollo de la competencia genérica de elaborar artículos científicos.

Palabras clave: competencias transversales; estudiantes en ciencias de la salud; artículos científicos.

\section{Summary}

The Journal of Medicine and Movies was always open to publish articles by students of health sciences and other degrees. Encouraging the participation of students was always one of the pretensions of the journal. The objective of this article is to describe the participation of students in the journal informing of the number of articles published, the number of authors, the type of work carried out, the universities of origin, the commented films and the topics discussed.

All articles published since 2005, at the beginning of the magazine, until 2019 were reviewed. 42 articles were registered with the participation of students $(16,9 \%$ of total) with 60 authors from 12 different universities and 10 different degrees, six of them from health sciences. 


\section{LA REVISTA DE MEDICINA Y CINE COMO FACILITADOR DE PUBLICACIONES CIENTÍFICAS DE LOS ESTUDIANTES JORGE PÉREZ; MARÍA JOSÉ FRESNADILLO; JOSÉ ELÍAS GARCÍA-SÁNCHEZ; ENRIQUE GARCÍA-SANCHEZ}

It is concluded that the Journal of Medicine and Movies, in addition to being a means to promote learning in health science studies, can facilitate to the students the development of the generic competence to produce scientific articles.

Key words: transversal competences; health sciences students; scientific articles.

\section{Introducción}

Durante el año 2019 se celebró el decimoquinto aniversario de la Revista Medicina y Cine considerable plataforma para fomentar las humanidades, especialmente a través del cine, en estudios en ciencias de la salud o en la actividad de los profesionales de todas sus disciplinas ${ }^{1,2}$.

En el Espacio Europeo de Educación Superior (EEES) se enfatiza en la adquisición de competencias genéricas por los alumnos, entre las más importantes se encuentran la de capacidad de expresión oral y escrita ${ }^{3}$. Así, la realización de artículos científicos por parte de los estudiantes sería una forma de mejorar dicha competencia. Hace algunos años, se apuntaba en un editorial del gran papel que podría jugar la Revista de Medicina y Cine para que los alumnos de estudios en ciencias de la salud pudieran desarrollar la competencia de escribir artículos científicos ${ }^{4}$.

El objetivo del presente artículo consiste en describir y comentar la situación sobre la autoría de artículos publicados en la Revista de Medicina y Cine de estudiantes de carreras diversas, especialmente de ciencias de la salud.

\section{Método}

Hemos seleccionado todos aquellos artículos, dentro de la sección de originales, en los que alguno de sus autores era estudiante en el momento de su publicación. El periodo registrado ha ido desde el inicio de la revista en 2005 hasta 2919, año del decimoquinto aniversario.

\section{Resultados}

En la revista, durante el periodo revisado, se publicaron 249 artículos en la sección de originales y en 42 de ellos, el $16,9 \%$, alguno de sus autores era estudiante en el momento de su publicación. De éstos, 27 hacían referencia a temas de salud, tanto a trastornos específicos como a otros aspectos, 10 analizaban películas concretas de interés sanitario, 2 comentaban obras literarias, 2 fueron editoriales y 1 explicaba una actividad académica sobre cine-fórum.

En los 42 artículos, aparecieron 60 autores que eran estudiantes, 24 de los cuales fueron varones y 36 eran mujeres, procedentes de 12 universidades diferentes (Tabla 1). Una estudiante participó en 4 artículos y 2 varones fueron autores en dos artículos.
Desde el punto de vista cinematográfico, en los artículos revisados se comentaron 45 películas diferentes, de las cuales 3 de ellas aparecieron en más de una ocasión. Así, El aceite de la vida / Lorenzo's Oil (1992) de George Miller fue comentada en tres ocasiones y tanto Philadelphia (1993) de Jonathan Demme como Gattaca (1997) de Andrew Niccol aparecieron en dos artículos diferentes. También la película de La Mosca / The Fly se comentó en dos ocasiones, pero haciendo referencia a sus dos versiones diferentes, la de 1958 y la de 1986, de Kurt Neumann y David Cronenberg respectivamente.

Los estudios que cursaban los autores en el momento de publicar sus artículos fueron muy diversos si bien predominaban los de ciencias de la salud, especialmente de Medicina. En concreto existieron autores estudiando 11 titulaciones diferentes (Tabla 1).

\section{Discusión}

Los datos de nuestro estudio indican que la implicación de alumnos en los artículos originales fue considerable ya que participaron en cerca del $17 \%$ de los mismos. Queremos resaltar que, al margen de la información descrita, centrada en la sección de originales, 2 estudiantes, un varón y una mujer, estuvieron vinculados a la revista participando de forma habitual en la sección de Medicina en Fotogramas.

En referencia al sexo de los autores, vimos que el porcentaje de mujeres (60\%) fue claramente superior al de varones (40\%). Dicha diferencia no nos puede extrañar ya que está en consonancia con la proporción de hombres y mujeres que actualmente se da en carreras de ciencias de la salud. Ello indicaría que no existirían deferencias de género en la implicación de publicar artículos científicos. Este hecho está en consonancia con otro estudio anterior, con estudiantes de ciencias de la salud, donde no encontramos diferencias de rendimiento académico entre hombres y mujeres 5 .

Hemos de destacar que los autores estudiantes procedían de 12 universidades diferentes. Si bien un porcentaje importante estudiaba en la Universidad de Salamanca, donde se edita la revista, se encontraron autores de otras universidades españolas y de tres de países hermanos latinos: México, Perú y Bolivia. Este dato indicaría una importante expansión y difusión de la revista.

En los artículos revisados fueron comentadas un número muy considerable de películas, en concreto 45 , pero solo tres de ellas aparecieron más de una ocasión: 
LA REVISTA DE MEDICINA Y CINE COMO FACILITADOR DE PUBLICACIONES CIENTÍFICAS DE LOS ESTUDIANTES JORGE PÉREZ; MARÍA JOSÉ FRESNADILLO; JOSÉ ELÍAS GARCÍA-SÁNCHEZ; ENRIQUE GARCÍA-SANCHEZ

Tabla 1. Procedencia de los estudiantes autores.

\begin{tabular}{|l|l|l|l|}
\hline \multicolumn{1}{|c|}{ Universidades } & \multicolumn{1}{c|}{ No de estudiantes } & \multicolumn{1}{c|}{ Nitulaciones de estudiantes } \\
\hline U. Salamanca (España) & 21 & Medicina & 33 \\
\hline U. Marista (Mexico) & 17 & Farmacia & 10 \\
\hline U. Pompeu Fabra (España) & 8 & Biología & 7 \\
\hline U. Norbert Wiener (Perú) & 4 & Enfermería & 3 \\
\hline U. País Vasco (España) & 3 & Arqueología & 1 \\
\hline U. Complutense (España) & 1 & Biomedicina & 1 \\
\hline U. Granada (España) & 1 & Comunicación & 1 \\
\hline U. Lleida (España) & 1 & Fisioterapia & 1 \\
\hline U. Málaga (España) & 1 & Humanidades & 1 \\
\hline U. Pablo de Olavide (España) & 1 & Traducción & 1 \\
\hline U. Pontificia de Salamanca (España) & 1 & No consta & 1 \\
\hline U. Salesiana (Bolivia) & 1 & & 1 \\
\hline
\end{tabular}

El aceite de la vida (1992), Philadelphia (1993) y Gattaca (1997). Precisamente estos tres films son muy utilizados y recomendados en la enseñanza de estudios de ciencias de la salud o de la bioética ${ }^{6,7}$.

En referencia a las carreras que estaban cursando los estudiantes autores, también hemos comprobado su diversidad. Si bien la mayoría cursaban estudios de ciencias de la salud, especialmente Medicina, se encontraron artículos de estudiantes de otras disciplinas no sanitarias como Arqueología, Comunicación, Humanidades o Traducción.

Resumiendo, podemos constatar una importante participación de alumnos en los artículos publicados en la revista y una procedencia muy variada tanto de universidades como de carreras. Ello estaría en consonancia con el objetivo de los editores de la revista de estimular la participación de estudiantes en la elaboración de artículos.

Como conclusión, consideramos que la Revista de Medicina y Cine puede ser una buena plataforma para que estudiantes, especialmente de ciencias de la salud, puedan publicar artículos relacionados con los objetivos de la revista. Ello contribuiría a estimular la competencia transversal de la realización de artículos científicos.

\section{Referencias}

1. García Sánchez JE, García Sánchez E. Hacia la segunda década de la Revista de Medicina y Cine. Rev Med Cine [Internet]. 2014; 10(4): 155-6.

2. Pérez J. RMC, quince años fomentando las humanidades en ciencias de la salud. Rev Med Cine [Internet]. 2019; 15(4): 193-4.

3. González J, Wagenaar R, editores. Tuning Educational Structures in Europe. Informe final Fase 1. Bilbao: Universidad de Deusto; 2003.

4. Pérez J. La Revista de Medicina y Cine, un medio para fomentar el aprendizaje de estudiantes en ciencias de la Salud. Rev Med Cine [Internet] 2013; 9(4): 149-50.

5. Centeno NB, Rodríguez G, Moyano E, Girvent M, Pérez J. Efecto del sexo en el rendimiento académico de estudiantes de Biología biosanitaria de la Universidad Pompeu Fabra. FEM 2019; 22 (6): 269-72.

6. Ranquer [Internet]. Los Angeles, CA. The Best Medical Movies Based on True Stories.

7. Care [Internet]. London. Bioethics Top 10 Films.

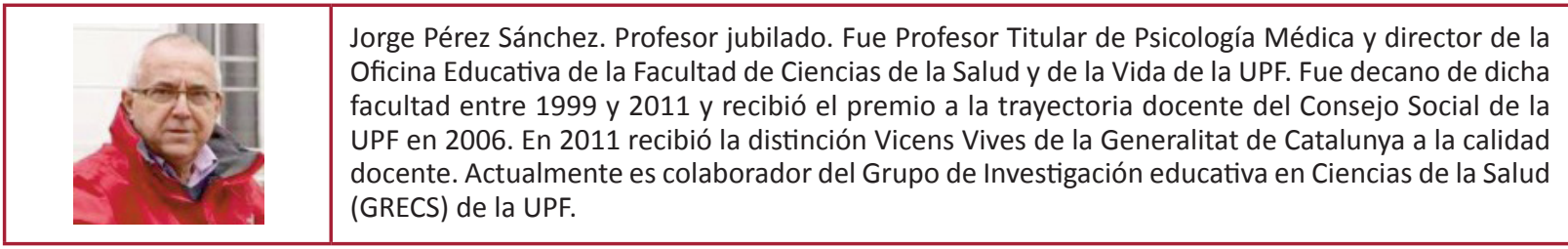

